\title{
Le discours sur la technologie aux États-Unis dans l'entre-deux-guerres (rencontre, mobilité, diffusion et construction)
}

Discourse about Technology in the interwar United States: Encounters, Mobility, Diffusion, and Construction

\section{Vincent Dray}

\section{(2) OpenEdition}

Journals

\section{Édition électronique}

URL : http://journals.openedition.org/artefact/687

DOI : 10.4000/artefact.687

ISSN : 2606-9245

Éditeur :

Association Artefact. Techniques histoire et sciences humaines, Presses universitaires du Midi

\section{Édition imprimée}

Date de publication : 15 juin 2017

Pagination : 127-138

ISBN : 978-2-7535-6525-8

ISSN : 2273-0753

\section{Référence électronique}

Vincent Dray, «Le discours sur la technologie aux États-Unis dans l'entre-deux-guerres (rencontre, mobilité, diffusion et construction) », Artefact [En ligne], 5| 2016, mis en ligne le 15 novembre 2017, consulté le 05 mars 2020. URL : http://journals.openedition.org/artefact/687 ; DOI : https://doi.org/ $10.4000 /$ artefact.687 


\section{Le discours sur la technologie aux États-Unis dans l'entre-deux-guerres (rencontre, mobilité, diffusion et construction)}

Vincent DRAY*

\section{Résumé}

L'article a pour objet l'analyse d'archives historiques relevant des positionnements conceptuels étatsuniens sur la technologie. L'émergence d'un concept en mouvement semble être la caractéristique du discours américain sur la technologie et restitué par les professeurs des instituts techniques. Afin de mettre en lumière la production des discours, la démarche ne peut laisser en marge les attentes d'une approche comparatiste mettant à l'étude quelques exemples européens dont l'influence est importante.

Mots-clés : études comparatives, instituts techniques, technologie.

\section{Abstract. Discourse about Technology in the interwar United States : Encounters, Mobility, Diffusion, and Construction}

Based on archival research in the US archives, this paper seeks grapple with US conceptual positioning with regards to technology. The emergence of the concept that is continually shifting seems one of the typical characteristics of the American discourse about technology developed by professors of Applied Science within Schools of Engineering. In order to shed

\footnotetext{
*. Chercheur à l'Institut de recherche sur les transports, l'énergie et la société (IRTES-RÉCITS), Vincent Dray travaille sur les mobilités internationales techniques et scientifiques transatlantiques. Parmi ses publications : "Démocratie technique et politique de la science au cours du second $x^{e}$ siècle ", in Yves Claude Lequin et Pierre Lamard (dir.), Éléments de démocratie technique, Belfort, université de technologie de Belfort-Montbéliard, 2014 et «La construction des échanges techniques franco-américains : sources, interdépendances et influences de 1914 à 1940 », Cahiers de RECITS, n 5, 2007, p. 175-197.
} 
light on the production of discourses about technology we will take a comparative approach highlighting some influential European examples.

Keywords : comparative studies, schools of engineering, technology.

Cet article a pour objet l'analyse de sources étasuniennes dont le centre d'intérêt est l'étude de la technologie. Il a pour thème central la construction d'un discours sur la technologie aux États-Unis, lequel est abordé à travers la circulation internationale des connaissances. Par combinaison, l'acquisition de systèmes technologiques étrangers, perçus ici comme des sources de compréhension des enjeux de la technique, peut apparaître comme un des éléments constitutifs mais non exclusif d'un discours et d'un cadre épistémologique. La méthode repose sur la recension puis l'analyse d'archives historiques relevant des positionnements conceptuels étatsuniens sur la technologie et pouvant indiquer les formes de sa légitimation, du point de vue des acteurs comme des institutions à l'intérieur desquelles elle se définit. Des travaux ont mis l'accent sur la technologie au concret dans le cadre de systèmes sociotechniques (Thomas Hughes), mais aussi sur la technologie en amont (David E. Nye ${ }^{1}$ ). Ces réflexions décrivent l'émergence d'un cadre épistémologique par lequel, aux États-Unis, le discours sur la technologie s'estémancipé d'une approche déterministe. Les outils bibliographiques montrent qu'au cours du premier $\mathrm{xx}^{\mathrm{e}}$ siècle, le terme Technology connaît aux États-Unis une mutation recouvrant des formes nouvelles de la modernité, lesquelles sont dégagées de définitions encombrantes et sont inspirées par la nécessité de saisir l'essor de la technologie dans une approche historique, sociale et culturelle ${ }^{2}$. L'émergence d'un concept en mouvement s'inscrivant dans l'histoire semble être la caractéristique majeure de l'évolution du discours américain sur la technologie et que restituent à l'époque, selon des cadres méthodologiques différenciés, les professeurs des Schools of Engineering, ainsi que les sociologues et historiens des grandes universités. En quelques années, les productions et travaux des instituts techniques et des départements des sciences sociales interrogent les rapports de la technologie à l'industrie, aux complexes militaires, à la ville (Chicago University), à l'éducation (Department of education : NARA) et au social. De ce point de vue, l'étude séminale de William F. Ogburn est très éclairante ${ }^{3}$. Ces productions révèlent l'évolution des réflexions sur le rapport triangulaire progrès/société/ technologie. Une rupture? De fait, une évolution. Car la machine-outil importée aux États-Unis au XIX siècle a façonné outre-Atlantique une représentation mécanicienne de la technique et de la machine, immortalisée, par exemple, par l'œuvre des photographes américains Paul Strand ${ }^{4}$, Alfred Stieglitz ${ }^{5}$ ou encore Berenice Abbott ${ }^{6}$.

Afin de mettre en lumière la production des discours, la démarche ne peut laisser en marge les attentes d'une approche comparatiste mettant à l'étude quelques exemples européens. Face à ce déploiement des sources, nous pouvons 
laisser la place à quelques hypothèses qui replaceraient l'histoire des techniques dans un cadre transnational en écho à l'internationalisation de la technologie. La démarche interroge ainsi le panorama des mobilités et des influences tant institutionnelles qu'humaines (émigrations scientifiques) qui se développent dans les milieux internationalistes de l'entre-deux-guerres.

Dans le contexte industriel qui s'imposait au tournant du siècle, le discours sur la technologie aux États-Unis a probablement pris en considération l'exigence économico-industrielle, mais a pu aussi interpréter la technologie comme une formulation de l'étude des techniques pour rendre ces dernières opérationnelles dans les champs de l'industrie. Autrement dit, la dimension sociale et culturelle a pris sa place dans la définition des termes. Cependant, l'existence de cette double approche explique qu'au cours du $\mathrm{xx}^{\mathrm{e}}$ siècle, le terme " technologie » a connu une définition différente par rapport au sens qui était le sien en Europe. D'ailleurs, du point de vue européen le sens étatsunien a longtemps revêtu presque exclusivement celui de l'innovation avant d'être associé à une science sociale, dimension que les sociologues et historiens des techniques, de Lewis Mumford et William F. Ogburn à Thomas Hughes, ont pu élaborer et qu'ils ont associée à la diversité des champs d'études de l'histoire des techniques.

Dans le cadre de l'analyse, il est remarquable que le discours sur la technologie se constitue aussi à partir d'un transfert de connaissances, lequel est révélé dans les études comparatives (comparative studies) réalisées en particulier dans les milieux universitaires au cours de l'entre-deux-guerres, période charnière des échanges technologiques, scientifiques et industriels entre les communautés techniciennes européennes et les États-Unis ${ }^{7}$. Ces acquisitions se réalisent dans le cadre de l'internationalisation des sciences et des techniques et révèlent l'influence européenne dans le contexte d'une représentation plus massive et plus précise de l'information technologique. Il s'agit bien d'un regroupement de connaissances faisant œuvre d'étude sur la technique (c'est le sens que nous donnons à l'internationalisation de la technologie). À ce contexte répond la construction d'un réseau entre universités et organismes gouvernementaux de recherche (National Research Council [désormais $\mathrm{NRC}$ ]) pouvant répondre à la demande d'une expertise scientifique plus poussée, en particulier à la suite du déclenchement du conflit européen, lequel est suivi de programmes plus soutenus faisant état d'une perspective internationaliste et technicienne (programme Wilson).

Nous voulons avancer qu'au cours de l'entre-deux-guerres se construit aux États-Unis un discours sur la technologie, lequel est élaboré en partie à travers un corpus de productions technologiques orienté et réalisé par les communautés techniciennes et scientifiques américaines et construit autour d'un intérêt pour l'étude comparée de systèmes éducatifs européens. La technologie est analysée ici dans le cadre de la circulation des connaissances. Cela explique l'état de la production de sources que l'on peut lier entre elles par des éléments de contextualisation, lesquels impulsent en retour la rédaction d'une littérature technologique. Mais quel est alors le 
contenu du concept de technologie tel qu'il se développe aux États-Unis et qui en partie s'appuie sur l'influence des modèles européens? Comment peut-on déterminer ce concept?

\section{Production : la fabrique des sources}

En termes de contexte, on fait ici référence à l'internationalisation des sciences et des technologies comme moyen de saisir les cheminements opérationnels de la technique. L'internationalisation des technologies est perçue ici à travers la masse d'une production d'informations qui décrit des postulats proposant la lecture de la technologie comme moyen d'interpréter la technique. Aussi, la technologie est ici une information qui se conforme à une production de sources. Cela reflète une donnée essentielle de la période de l'entre-deux-guerres : le rôle incontournable de la documentation technique et industrielle.

Les sources que nous présentons sont donc du point de vue historique des productions technologiques. Le contexte de la production des sources révèle une symétrie avec celui de l'internationalisation des technologies, laquelle est impulsée par les milieux universitaires internationalistes que $l^{\prime}$ on retrouve dans ceux des élites techniciennes, scientifiques, ou encore dans les milieux de l'art photographique $^{8}$. L'internationalisation des technologies, face à un marché en concurrence, se décline en de multiples mouvements internationalistes qui s'organisent au sein de nombreuses branches des sciences sociales et des arts. Mais c'est la concurrence des marchés et singulièrement celle des marchés de la technique industrielle qui peut appa- raître comme un facteur déterminant du développement des programmes technologiques assurant la production des sources d'informations et de connaissances. Les marchés internationaux des secteurs innovants laissent place à un cycle de productions technologiques intégrant une massification de l'information. Ainsi, apparaît un des points contextuels autour duquel s'articulent les politiques économiques et techniques de la période de l'entre-deux-guerres, lesquelles politiques sont devenues des enjeux incontournables de la puissance.

Une autre caractéristique de la catégorie des sources étatsuniennes analysées tient au fait qu'elles ont pour objet l'étude des environnements technicoindustriels des systèmes étrangers. On peut avancer que le discours des élites techniciennes étatsuniennes sur la technologie, tel qu'il se construit au cours de l'entre-deux-guerres est le fait d'une combinaison entre une culture technique nationale et l'apport de ces systèmes, lesquels incluent un usage fort différencié de la technologie comme science sociale. Il est évident que le contexte de la guerre explique les orientations géographiques des études comparatives étatsuniennes. Si l'Allemagne apparaît comme un modèle d'influence pour les États-Unis, c'est en revanche la France qui intéresse davantage les milieux technico-scientifiques. L'intérêt pour la France peut 
être replacé dans un contexte plus large, celui de la compétition, dont l'objet est la technologie, et plus largement de la compétition qui s'ordonne autour de l'Allemagne qui pour les États-Unis est à la fois concurrente et partenaire. Et si la France intéresse davantage les ÉtatsUnis c'est bien parce que le système est celui qui est le plus éloigné du système outre-Atlantique et probablement le plus proche de la fonction originelle de la technologie, c'est-à-dire d'être objet d'étude de la technique et donc une catégorie des sciences sociales. Pour les ingénieurs français, la technologie est le moyen d'amener la technique dans ses cheminements opérationnels et dans ses usages. C'est pourquoi la France est représentée à travers la place que prend la culture générale dans les instituts techniques et au sein des grandes écoles d'ingénieurs. Entendons par culture générale l'option consistant à donner une dimension historique à la technique et c'est peut-être dans l'enseignement de la culture générale que la technologie acquiert une dimension sociale en France. Ce ne sont pas nécessairement les systèmes d'éducation qui intéressent le plus mais plutôt la manière dont est intégrée la technologie. Ce corpus de sources américaines est de notre point de vue représentatif d'une production qui constitue une des bases de l'exploration de la technologie comme science sociale. Aussi, ces facteurs d'analyse révèlent la réalité d'un transfert de connaissance dont les points majeurs structurent, au moins partiellement, et non pas exclusivement, le discours américain.

\section{Mobilité : échanges et acheminement}

Les professeurs des instituts universitaires de la côte est des États-Unis sont très impliqués dans les questions du développement et de l'évolution de l'enseignement technique. Mais, en premier lieu, leur enseignement recouvre celui des sciences appliquées industrielles. C'est en partie ce qui justifie les recherches des professeurs des schools of engineering des instituts techniques universitaires sur les enjeux des sciences industrielles appliquées et leurs relations avec l'industrie. À la base de ces études, une réflexion sur le système éducatif technique et scientifique moderne ${ }^{9}$. $C^{\prime}$ est à travers une réflexion sur les sciences industrielles que l'intérêt des Américains pour les systèmes éduca- tifs étrangers s'est développé ${ }^{10}$. On peut ainsi rendre compte du statut analytique et de la légitimité historique d'un corpus de sources d'archives et imprimées qui pense la technique. Mais néanmoins on peut aussi faire ressortir les différentes articulations conceptuelles et contextuelles qui distinguent la technologie comme science industrielle appliquée, interprétée comme telle dans les milieux des instituts techniques, et la technologie comme objet de sciences sociales telle qu'elle est interprétée dans les départements de sciences sociales. Peut-on déceler les articulations qui permettront au cours de la période d'impulser un rapprochement des deux dimensions dans un discours construit sur la technologie, 
alors même que les productions technologiques analysées sont la production des professeurs des instituts techniques? L'approche humaniste des professeurs de sciences suffit-elle à expliquer l'évolution du terme technologie, qui ne serait plus seulement réductible aux sciences industrielles mais qui au contraire s'établirait comme objet des sciences sociales et objet d'étude de la technique?

En 1918, les États-Unis n'avaient fixé ni leurs choix ni leurs stratégies techniques dans l'ensemble des secteurs. Ce panorama général peut s'illustrer à partir de la répartition par pays des Prix Nobel de physique et de chimie obtenus durant la période. Les États-Unis occupent le seuil le plus bas jusque dans les années 1930, période durant laquelle ils dépassent la France. Enfin, c'est seulement après la Seconde Guerre mondiale que les États-Unis surpassent l'ensemble des pays européens ${ }^{11}$. Ce n'est qu'à partir des années 1920 que les États-Unis regroupent les éléments structurels qui permettront de faire émerger à grande échelle la recherche industrielle grâce à l'éclosion des grands laboratoires de $R \& D$. Pour se lancer dans la recherche à grande échelle, il fallut un cadre institutionnel. C'est dans ce contexte que fut créé le NRC en $1916^{12}$. On notera que certains des ingénieurs qui portèrent un intérêt à l'Europe sont aussi ceux qui s'intéressèrent aux instituts techniques allemand et français, en particulier Arthur Edward Kennelly, professeur au MIT et membre de l'American Institute of electrical engineers (AIEE ${ }^{13}$ ).

On se situe bien dans le contexte de l'internationalisation des technologies où s'opère un rapprochement institutionnel. L'aspect technique, déterminant dans la progression des secteurs, est lié à un ensemble de relations qui se mirent en place entre des acteurs publics et privés ou entre différentes institutions, ce que $l^{\prime}$ analyse politique a mis en avant ${ }^{14}$. Cette transformation institutionnelle était en relation avec d'importantes mutations intellectuelles concernant notamment le regard sur la science. Dans le dernier tiers du xIX ${ }^{\mathrm{e}}$ siècle, les relations entre les scientifiques et les techniciens devinrent plus étroites dans le monde universitaire ${ }^{15}$, ce qui accompagnait les progrès réalisés dans la formation des ingénieurs et cadres techniciens ${ }^{16}$. La puissance américaine trouve ses origines dans la capacité à mobiliser les connaissances dans des cadres institutionnels et donc à développer des courants de pensée et des communautés d'intérêt pouvant acheminer les connaissances scientifiques vers le développement industriel et commercial.

Les études et analyses portant sur la France proviennent d'une démarche multidisciplinaire américaine qui regroupe au sein de la Society for American fellowship in French universities un très important nombre d'auteurs et collaborateurs universitaires, plus de cent au total, dans le cadre d'une publication portant sur l'environnement universitaire et l'enseignement en France et titrée Science and learning in France ${ }^{17}$. Quelques-uns de ces professeurs étaient connus : L. H. Henderson (Harvard University) dans le domaine de la chimie, Frederic E. Farrington dans le domaine de l'éducation, Henry M. Howe de l'université de Columbia, Alex C. Humphreys du Stevens Institute of technology, Albert Sauveur de l'université d'Harvard. Cette étude met en lumière les moyens utilisés 
pour diffuser une vision de l'utilité des sciences. Rappelons que le président de l'American manufacturers export Association encourage au même moment aux relations interuniversitaires afin de développer les contacts commerciaux et industriels.

Après la guerre, on assista à la mise en place d'échanges universitaires de plus en plus spécifiques, organisés et dirigés vers des branches liées aux sciences industrielles ${ }^{18}$. Ces relations furent possibles grâce à des accords conclus entre les universités américaines et le ministère français en charge de l'éducation. Les rapports annuels des universités américaines confirment le suivi des échanges entre des universités de la côte est, des instituts techniques français et des universités françaises, $\mathrm{du}$ moins $\mathrm{d}$ 'une manière régulière jusqu'en 1925. Du côté américain, les échanges font intervenir des professeurs des schools of engineering; ils écrivent dans les revues spécialisées, telle que Engineering education ou Bulletin of investigation. Ils se nomment Arthur Edward Kennelly ${ }^{19}$, Dugald Caleb Jackson ou William E. Wickenden. En outre, ils sont membres de l'American Institute of education, de la National Academy of science, du NRC et membres actifs de l'AIEE. Dans un cadre technique international, ils se rattachent à l'International electrotechnical Commission (IEC). Aux États-Unis, le choix des modalités d'échange avec la France fut laissé à l'initiative des professeurs des schools of engineering. C'est pourquoi, on peut disposer de sources intéressantes avec les publications des professeurs américains et les rapports et programmes disponibles dans les archives universitaires.
Les archives et les rapports annuels des universités américaines de Columbia et d'Harvard, auxquels il faut ajouter celles et ceux du MIT, rapportent l'existence de relations scientifiques et techniques officielles entre les États-Unis et la France. Elles engendrent des échanges de professeurs ou d'instructeurs. En 1919, des accords furent conclus entre le ministère français de l'Instruction publique et l'administration de sept institutions universitaires américaines de la côte Est qui, à cette occasion, mirent en place un comité général : Columbia, Cornell, Harvard, Johns Hopkins, MIT, université de Pennsylvanie, Yale. Ces universités entretinrent des relations avec des universités françaises, mais aussi avec des instituts techniques indépendants et des grandes écoles, en particulier l'École centrale des arts et manufactures ${ }^{20}$. Arthur Edwin Kennelly, professeur d'électricité appliquée à l'université d'Harvard et inventeur réputé, et Dugald Caleb Jackson, lui aussi professeur d'électricité appliquée, sont membres du comité qui coordonne les échanges franco-américains $^{21}$. Il s'agissait d'un plan d'échange de professeurs des sciences appliquées et de technologie ${ }^{22}$. Ce plan était destiné à développer les échanges en sciences appliquées et en science de l'ingénieur. Deux aspects sont à souligner : le caractère technique porté par les sections technologiques des universités américaines; le caractère organisé et institutionnalisé des échanges, d'abord développés, du côté américain, par l'intermédiaire d'organismes nationaux et internationaux. C'est en effet dans ce cadre que peuvent réellement jouer les influences des organismes techniques. Les accords avaient été menés du côté américain par 
l'Institute of international education et par l'AIEE, organismes composés de certains professeurs américains qui participèrent à ces échanges.

\section{Réception : construction du discours et cadre épistémologique}

Le point majeur porte sur le sens associé au terme technologie. Comment en effet le concept de technologie devientil aux États-Unis la matrice d'une science sociale de la technique? Peut-on parler d'une différence avec la définition de la technique comme science appliquée? Depuis la guerre, les belligérants avaient cherché à améliorer leur système éducatif et les enseignements dispensés dans les cours d'enseignement des techniques. Les États-Unis avaient aussi cherché à définir des politiques sur les finalités de ces enseignements. À la fin des années 1920, il fallait en tirer les conséquences mais aussi des expériences. William E. Wickenden, spécialiste des sciences appliquées, est un personnage connu du monde de la recherche ${ }^{23}$. Il est un témoin des principales transformations de l'enseignement des techniques industrielles. À deux reprises, en 1925 et 1928, ce professeur voyage en Europe afin d'observer les caractéristiques de l'enseignement technique et scientifique de chacun des principaux pays industrialisés objets de ses études. Un an après son dernier voyage, en 1929, il publie une importante étude comparative sur les systèmes d'enseignement technique de la France, de l'Allemagne, de l'Angleterre et pour finir des États-Unis ${ }^{24}$. On peut supposer que les influences et les échanges institutionnalisés qui se sont opérés depuis le début du siècle expliquent, justifient et donnent une matière d'analyse à ces études comparatives, comme pour justifier la construction d'un processus qui aura permis aux États-Unis de construire un « modèle technique ». Une véritable explication des transferts de connaissances et de technologies de l'Europe vers les États-Unis est mise en avant par les observateurs et vient $s$ 'instituer dans l'internationalisation des sciences ${ }^{25}$. Cette remarque justifie à elle seule, de manière structurelle, l'existence des études comparatives et surtout leur utilisation comme sources d'archives. Elles reposent sur une interprétation des systèmes éducatifs et surtout sur la recherche de similitudes institutionnelles avec les pays européens industrialisés. Le second angle est marqué par la nécessité de trouver des répondants afin d'engendrer et perpétuer des coopérations universitaires qui auront pour effet, dans les perspectives américaines, de rebondir sur la multinationalisation des techniques et d'orienter la coopération technologique. Si le processus n'est pas abouti, comme il le sera dans les années 1960, il n'en reste pas moins vrai que nous nous situons à la fin des années 1920, dans une période charnière du processus d'échanges et d'influences réciproques. C'est probablement à cette époque que la tendance s'inverse réelle- 
ment et que le transfert et l'influence de l'Europe vers les États-Unis devient un enjeu et entre dans sa dernière phase, celle de l'assimilation et de la réception.

Concernant l'enseignement technique, les ingénieurs scientifiques américains donnèrent très tôt un sens concret et pratique à leurs études comparatives tout en prenant en considération les variations de sens du vocabulaire en fonction des pays, surtout dans les domaines spécifiquement industriels et dans les branches qui, aux États-Unis, furent très tôt considérées comme des noyaux durs de l'industrie moderne. Tel que cela est clairement exprimé dans les comparative etudies américaines, les terminologies présentaient des difficultés d'interprétation pour les experts. Le vocabulaire technique employé pour décrire les institutions techniques américaines différait du vocabulaire européen comme le soulignent quelques études ${ }^{26}$. Les rapports publiés par 1'American Society for engineering education et par la Society for the promotion of engineering education (Wickenden) soulignent les problématiques que soulèvent les variantes terminologiques et les différentes mises en œuvre opérationnelles que cela conduit à prendre en considération dans le domaine de l'enseignement technique. D'une manière générale, il était donc difficile d'entreprendre une analyse comparative et de trouver des termes ayant une commune acception. Il est vrai que le terme " engineering education » est utilisé de manière approximative aux États-Unis et n'a pas d'équivalent direct dans la terminologie européenne. Les sources distinguent un système continental. Sur le continent, l'équivalent du terme "engineering education » est «technical education », terme qui désignait la formation technique dans toutes les branches. En Angleterre, le terme désignait l'éducation spécialisée concernant les machines-outils. De plus, les nomenclatures employées en Europe occidentale comme " higher ", " middle » et «lower » sont utilisées pour faire la distinction entre plusieurs types d'école d'enseignement technique. Aux ÉtatsUnis, ces catégories sont alors employées pour distinguer des "degrés d'excellence ». Cette difficulté à décrire l'enseignement technique français traduit une inadéquation entre les systèmes.

C'est que l'approche technologique de l'enseignement ne porte pas exclusivement sur les sciences appliquées mais sur la conception même de l'enseignement et sur la description de la technique. Les observateurs soulignent que ce qui fait la distinction entre un ingénieur français et un ingénieur américain est probablement le bagage social et culturel que le premier inscrit dans la représentation et l'histoire de la pensée technique. Dans une adresse délivrée à des étudiants de l'université de Purdue, où il est professeur assistant et enseigne l'électricité appliquée, Alfred Still compare les systèmes éducatifs destinés aux ingénieurs en Europe et aux États-Unis ${ }^{27}$. Datant de 1915, son article n'en reste pas moins explicite et est à porter au crédit de la période de l'entre-deux-guerres. La première remarque, et cela est valable pour l'Angleterre, la France et l'Allemagne, est que le niveau général d'éducation à l'entrée à l'université y est plus élevé qu'aux États-Unis, cela n'étant pas sans rappeler que l'enseignement technique européen est basé sur l'importance de la culture générale, c'est-à-dire l'introduction de l'art de la technique dans 
une dimension historique, comme produit de l'activité humaine, comme objet de société. Alfred Still rappelait que les ingénieurs des écoles européennes, plus que ceux des universités américaines, disposaient d'une plus large originalité, d'une imagination plus vive et de plus grandes potentialités de création pouvant combiner entre eux les éléments mis à leur disposition. L'ingénieur doit être pratique (practical man) et disposer d'une grande culture générale, d'une connaissance des choses matérielles, d'une connaissance littéraire.

Ces travaux comparatistes sont d'autant plus pertinents qu'ils sont produits par des professeurs qui avaient vécu des expériences professionnelles en Europe, ce qui est le cas d'Alfred Still qui enseigna pendant longtemps en Angleterre et qui émigra aux États-Unis pour y faire sa carrière $d$ 'ingénieur scientifique dans le domaine des transports de courant.

Même si dans les universités américaines et européennes, dans le contexte de la concurrence, la tendance à laisser de côté les matières non techniques se généralisait, cela ne traduisait qu'une tendance à la spécialisation. Mais cette tendance révélait aussi les formes innovantes d'une reformulation de l'enseignement des techniques industrielles et de son cheminement vers des études technologiques, lesquelles avaient pour fonction d'intégrer une dimension sociale et culturelle pouvant expliquer leur développement. Les professeurs des instituts techniques semblent avoir pris en compte les évolutions constatées en Europe. Sans avancer que l'introduction de la culture générale dans l'enseignement technique au sein des instituts américains soit un facteur exclusif de conceptualisation du terme technology, les observateurs américains, en présentant les enjeux et articulations de cette culture générale dans le cadre de l'enseignement des techniques, ont contribué à redéfinir le sens même de la technique dans la société et à développer un cadre épistémologique relayé concrètement au cours des années 1930 par les départements de sciences sociales (université de Chicago, université de Columbia). Au fond, cette littérature grise des instituts techniques est aussi le ressort d'un cadre épistémologique que sociologues et historiens outre-Atlantique chercheront à développer à leur tour en s'appuyant sur les éléments forts des critiques portées à l'endroit de la technique, surtout en Europe. La contribution des professeurs d'instituts techniques s'est cristallisée autour de publications, de conférences, d'ouvrages collectifs et surtout elle s'est organisée par l'implication dans des réseaux nationaux et internationalistes encouragés par les universités et les organismes gouvernementaux. Cela se ressent encore au milieu des années 1930 où les experts présentent des discours à des associations de professionnels ${ }^{28}$. 


\section{Notes}

1. David E. NYE, Technology matters. Questions to live with, Cambridge, The MIT Press, 2006.

2. On peut s'appuyer sur un travail récent, Eric Schatzbergs, «Technik comes to America: changing meanings of technology before 1930 ", Technology and culture, vol. 47, $\mathrm{n}^{\circ}$ 3, July 2006, p. 486-512.

3. William F. Ogburn, «Technological trends and national policy, including the social implications of new inventions. Report of the subcommittee on technology to the National Resources Committee ", United States Government printing office, Washington, juin 1937.

4. Paul Strand, sixty years of photographs, profile by Calvin TomKins, An Aperture Monograph, 1976. Voir en particulier la série photographique des machines-outils (Lathe; Drilling Machine; Akeley Camera; Wire Wheel).

5. Alfred Stieglitz, The hand of Man, Long Island City, NY, 1902, photogravure, 8 3/4 in. x 6 $5 / 8$ in. (Colby College Museum of Art), published in Camera Work, 1, January 1903.

6. Bonnie Yochelson, Berenice Abbott, changing New York. The complete WPA Project, New York, The Museum of the city of New York, 1997.

7. Vincent DraY, Dans les mouvements de la modernité : la vision de l'Autre. Interdépendances et influences technologiques entre les États-Unis et la France de 1914 au milieu des années 1930, thèse de doctorat sous la direction d'Albert Broder, Paris Est, Créteil, 2008.

8. Mitra Авbaspour, Lee Ann Daffner, Maria Morris Hambours (dir.), Modern photographs, the Thomas Walther Collection, 1909, 1949, New York, Moma, 2015.

9. Dugald Caleb JACKson, "Co-operation between the technical industries and technical education in America ", in The Institute of electrical enginneers of Japan, Tokyo, octobre 1935.

10. Voir, par exemple, à la New York Public Library (NYPL), Maj. William D. ConNor, National School of bridges and highways, 'L'École nationale des Ponts et Chaussées' of the Corps des Ponts et Chaussées, Washington, 1913; à l'université de Columbia (School of Engineering Library), William E. WicKeNDEN, A comparative study of engineering education in the United States and in Europe, Bulletin number 16 of the Investigation of engineering education, New York, Library of School engineering of Columbia, juin 1929.

11. Richard R. Nelson, Gavin Wright, "The rise and fall of American technological leadership », Technology and culture, p. 1941 (graphisme : cumulative Nobel Prize in physics and chemistry, 1901-1990).

12. La structure fut inspirée du Physikalisch Technishe Reichsanstalt, devenu, après 1918, le Max Plank Gesellschaft. David CAHAn, An institute for an empire. The Physikalisch-Technische Reichsanstalt, 1871-1918, New York, Cambridge University Press, 1989, p. 3.

13. Sur Arthur Edward KenNelly et ses notes sur la physique, Henry S. CARHART, « The imperial physico-technical institutions in Charlottenburg ", Transactions of the American Institute of electrical engineers, $\mathrm{n}^{\circ} 17,1900$, p. 555-583, incluant les remarques p. 582 sur le Reichsanstalt par A. E. Kennelly, source citée par D. CAHAN, in An institute for an empire, op. cit., p. 229.

14. Louis Galambos, Joseph Pratt, The rise of the corporate Commonwealth: United States business and public policy in the $20^{\text {th }}$ century, New York, Basc Book, 1988.

15. David F. Noble, America by design, science, technology and the rise of corporate capitalism, New York, Oxford University Press, 1977.

16. Daniel E. Alexander, The development of engineering education in the USA, Washington State University, 1977; D. Noble, America by desing..., op. cit., chap. 8 .

17. John H. Wigmore (dir.), Science and learning in France, with a survey of opportunities for American students in French universities, an appreciation by American scholars, New York, Columbia University, The Society for American fellowships in French universities, 1917.

18. V. Dray, Dans les mouvements de la modernité, op. cit.

19. Arthur E. Kennelly, « On the educational engineering in France », Publication of the MIT, Electrical engineering department, Research Division Bulletin, $n^{\circ} 32,1923$, p. 89-118.

20. Massachusetts Institute of technology Archives [ensuite MIT Archives], Annual Report, TC1, années 1919-1920, 1921.

21. Arthur Edwin Kennelly se rendit en France en 1921-1922. Il présenta ses travaux dans les écoles électrotechniques et les universités, mais aussi devant un auditoire de l'École professionnelle des postes et télégraphes. L'ingénieur et professeur américain rencontra Paul Janet, directeur de l'École supérieure d'électricité, et donna des conférences devant les étudiants de l'établissement parisien. D'autre part, il se rendit au Laboratoire central d'électricité. MIT Archives, MC 328: Arthur Edward Kennelly; Harvard University Archives, HUG 1479.80.4, Memories of Arthur Edwin Kennelly. 
22. Columbia University Archives, Annual Report, Dean of the School Engineering, 1921, 1922, p 122-123.

23. President, Case School of Applied Science, directeur de Investigation of engineering education (Society for the promotion of engineering education).

24. W. E. WiCKenden, A comparative study of engineering education in the United State and in Europe, op. cit. Cette étude est publiée de nouveau en 1934 : Society for the promotion of engineering education, Report of the investigation of engineering education 1923-1929, with Report On Summer Schools for engineering teachers, 1927-1929, University of Pittsburgh, 1934.

25. Vincent DrAY, « La diffusion internationale des technologies. La construction des échanges techniques franco-américains : sources, interdépendances et influences de 1914 à 1940 », Cahiers de RECITS, $\mathrm{n}^{\circ}$ 5, décembre 2007, p. 175-197.

26. « Report of the investigation of engineering education 1923-1929 », American Society for engineering education, vol. II, Pittsburgh, PA, 1934, p. 3.

27. Alfred STILL, « The engineering student in Europe and America ", Bulletin of the Society for the promotion of engineering education, $\mathrm{n}^{\circ} 10,1915$, p. 63-75.

28. W. E. WICKENDEN, « Effect of engineering schools on the social and economic life of Europe ", Journal of engineering education, $\mathrm{n}^{\circ} 24$, presented at the 40th anniversary meeting, SPEE, June 26-30, 1933, Chicago, septembre 1933, p. 190-199. 